\title{
Modelación del crecimiento de pollitas Lohmann LSL con redes neuronales y modelos de regresión no lineal
}

\section{Modeling of growth in Lohmann LSL pullets with neural networks and nonlinear regression models}

\author{
Luis Galeano-Vasco,* M.Sc, Mario Cerón-Muñoz, Ph.D. \\ Universidad de Antioquia, Facultad de Ciencias Agrarias, Grupo investigación Genética, Mejoramiento \\ y Modelación Animal, A.A. 1226, Medellín, Colombia. *Correspondencia: If.galeano.vasco@gmail.com
}

Recibido: Abril de 2012; Aceptado: Febrero de 2013.

\section{RESUMEN}

Objetivo. Modelar la curva del crecimiento de aves de la línea Lohmann LSL utilizando modelos no lineales (MNL), no lineales mixtos (MNLM) y redes neuronales artificiales (RNA). Materiales y métodos. Periódicamente se pesaron 33 aves en promedio, desde el día 21 al 196 de vida para un total de 558 registros individuales de peso. En el ajuste de la curva de crecimiento se utilizaron los modelos: no lineal de Von Bertalanffy (MNL), no lineal Mixto de Von Bertalanffy (MNLM) y redes neuronales artificiales (RNA). Los modelos se compararon con coeficiente de correlación y medidas de presicion cuadrado medio del error (CME), desviación media absoluta (MAD) y porcentaje de la media absoluta del error (MAPE). Resultados. Los valores de correlación entre los datos reales y estimados, fueron 0.999, 0.990 y 0.986 para MNLM, RNA y MNL respectivamente. El modelo más preciso con base en los criterios MAPE, MAD y CME fue el MNLM, seguido por la RNA. La grafica de predicción generada la RNA es similar a la del MNLM. La RNA presentó un desempeño superior al MLN. Conclusiones. El mejor modelo para la predicción de curvas de crecimiento de aves comerciales de la línea Lohmman LSL hasta los 196 días de edad, con múltiples mediciones por animal en el tiempo, fue el MNLM. La RNA presento un desempeño superior al MNL.

Palabras clave: Crecimiento, modelo no lineal, modelo no lineal mixto, redes neuronales artificiales (Fuente: MeSH).

\begin{abstract}
Objective. Modeling the pullet growth curve of the Lohmann LSL line, by using nonlinear model (MNL), nonlinear mixed model (MNLM) and artificial neural networks (ANN). Materials and methods. An average of 33 birds, were weighed from day 21 to 196 of life for 558 individual weight records. To adjust the growth curve the following models were used: nonlinear Von Bertalanffy (MNL), nonlinear mixed Von Bertalanffy (MNLM) and artificial neural networks (RNA). The models were compared with a correlation coefficient and precision measurements: mean square error (MSE), Mean Absolute Deviation (MAD) and the mean absolute percentage error (MAPE). Results. Correlation values, between actual and estimated data, were $0.999,0.990$ and 0.986 for MNLM, RNA and MNL respectively. The most accurate model based on the MAPE, MAD and CME criteria was MNLM followed by RNA. The prediction graph for RNA was similar to MNLM. The RNA performance was higher than MLN. Conclusions. The best model for the prediction of growth curves of commercial Lohmman LSL birds to 196 days of age, was the MNLM, with multiple measurements per animal at the time. RNA performance was higher MLN.
\end{abstract}

Key words: Connectionist Models, growth, Non-linear Models, nonlinear mixed effect model (Source: MeSH). 
3862 REVISTA MVZ CÓRDOBA • Volumen 18(3) Septiembre - Diciembre 2013

\section{INTRODUCCIÓN}

El rendimiento productivo de las aves de corral en la producción de huevo comercial, está asociado al desarrollo óseo, muscular y reproductivo alcanzado durante la fase de cría y levante (1, $2)$. En este periodo el objetivo del avicultor es obtener lotes de animales con un peso y uniformidad acorde a las exigencias de la casa genética, para alcanzar la madurez sexual a la edad optima para expresar todo su potencial productivo (3).

El desarrollo del ave en la etapa de cría y levante está condicionado por factores ambientales (temperatura, humedad relativa, velocidad del viento y luminosidad), de manejo, nutricionales, genéticos y de instalaciones (4, 5), entre otros. La medición, caracterización y modelación de estos factores favorece la implementación de acciones preventivas y correctivas tendientes a ofrecer a los animales un ambiente confortable, disminuyendo así las pérdidas productivas ocasionadas por los cambios en el comportamiento y gastos energéticos del metabolismo en respuesta a eventos o factores causantes de stress de las aves $(6,7)$.

En tal sentido, para la descripción ajuste y predicción de la curva de crecimiento en aves se han utilizado las funciones de Brody (8), Gompertz (9), Logística (10), Richards (11), Von Bertalanffy (12), modelos con la inclusión de algoritmos genéticos (13) y las RNA (14), entre otros.

La principal diferencia entre los MNL y MNLM es la inclusión de efectos aleatorios con el fin de explicar parte del error total a partir de la variabilidad individual. Por tal motivo los MNLM generan estimaciones que representan con más fidelidad el comportamiento de variables medidas en el tiempo al cuantificar la variación dentro y entre los individuos. El resultado final de un MNLM son los efectos fijos con los valores esperados y los efectos mixtos con la varianza y la covarianza de las observaciones (15).

Autores como Ahmadi et al (16) y Roush (14) han comparado la capacidad de ajuste de las RNA con otros modelos usando datos de crecimiento de pollos de engorde, encontrando que las RNA son más fáciles de usar y más eficientes; además como no tienen una ecuación prediseñada tienen la posibilidad de trabajar con datos que tengan "ruido" y así maximizar su poder de ajuste. En otra evaluación Yee (17), modelando el crecimiento de ovejas, afirmó que las RNA fueron menos influenciada por la variabilidad longitudinal en los datos lo que le da superioridad ante otros modelos por combinar la exactitud y precisión en el ajuste de la curva.

Otro aspecto es que son RNA son técnicas de distribución libre o no paramétricas, admitiendo la incorporación de todo tipo de datos independientemente del cumplimiento de los supuestos teóricos relativos a las técnicas estadísticas (normalidad, homocedasticidad, independencia, etc.) $(18,19)$. Pero lo más relevante de las RNA radica en su capacidad de aprender y reestructurarse a sí misma, convirtiéndola en un modelo que está en constante adaptación (20).

En este estudio se comparó la capacidad para modelar la curva de crecimiento (peso en gramos) de las redes neuronales, modelos no lineales y modelos no lineales mixtos. Este análisis se realizó como punto de partida para la construcción de un modelo basado en RNA que incorpore un número mayor de variables de entrada tales como: condiciones ambientales, genéticas, de manejo y nutricionales, entre otras; usadas para modelar la curva de crecimiento de aves destinadas a la producción de huevo comercial.

\section{MATERIALES Y MÉTODOS}

Tipo de estudio. Estudio longitudinal con diseño de medidas repetidas en el tiempo, donde la variable dependiente (peso del ave) es evaluada en función del tiempo (edad del ave) para cada individuo.

Sitio de estudio. La información utilizada para la modelación procede de un lote de aves de la Hacienda La Montaña, propiedad de la Universidad de Antioquia, localizada en el municipio de San Pedro de Los Milagros (Antioquia) a los $6^{\circ} 19^{\prime} 19^{\prime \prime}$ ' de latitud norte y a $1^{\circ} 37^{\prime} 40^{\prime}$ ' de longitud occidental. Con altura de 2.350 m.s.n.m. y temperatura promedio de $15^{\circ} \mathrm{C}$ (máxima $22^{\circ} \mathrm{C}$ y mínima $7^{\circ} \mathrm{C}$ ).

Muestra. Para la realización de la evaluación del crecimiento se utilizaron 100 aves de la línea Lohmann LSL seleccionadas al azar de un lote de gallinas ponedoras de huevo comercial.

Recolección de datos. La muestra inicial fue de 100 aves, las cuales el día 21 de vida fueron marcadas y pesadas. Desde la fecha de inicio de la evaluación hasta la edad de 196 días, las aves fueron evaluadas por 17 veces (cada $11 \pm 6$ días). Las pollitas terminaron su fase de cría y 
levante en piso el día 112, durante este periodo se recapturaron 26 aves en promedio en las 11 mediciones realizadas. A partir del traslado a las jaulas hasta cumplir 196 días de edad 35 aves fueron pesadas 6 veces. Los 65 animales faltantes no se encontraron al momento del traslado debido a pérdidas de las etiquetas de marcado en cría y levante o muerte. En total se obtuvieron 558 datos de pesaje para el ajuste de los modelos. Durante todo el proceso las aves recibieron alimento y agua fresca ad libitum, con base en los criterios propuestos por la línea genética y los requerimientos nutricionales propios del sistema de cría y levante en piso.

Análisis estadístico. Para el entrenamiento de la red se utilizaron 446 datos de pesaje (80\%). La estructura de la neurona se basó en el perceptron multicapa, con una neurona de entrada (edad del ave en días), seis neuronas en la capa oculta y una neurona de salida (peso del ave g.). La función de activación de las neuronas ocultas fue:

$$
f(x)=\operatorname{tansig}(x)=\left(\frac{2}{1+e^{-2 x}}\right)-1
$$

La RNA se programó para que la red pudiese generar relaciones lineales con la neurona de salida (linout=TRUE), con la posibilidad de crear conexiones directas entre la neurona de entrada y de salida (skip=TRUE) y con un mínimo de 1000 iteraciones (miniter $=1000$ ). La elección de estos parámetros fue llevada a cabo de forma heurística a través de ensayos pilotos en simulaciones previas.

Para la selección de los modelos estadísticos se realizaron ensayos previos en los cuales se comparó la capacidad de ajuste de los modelos Logístico, Brody, Richards, Gompertz y Von Bertalanffy, siendo este último el de mejor desempeño hasta los 196 días de edad del ave.

El modelo no lineal Von Bertalanffy (12) utilizado para el ajuste de la curva de crecimiento,

$$
y_{i j}=\beta_{0} *\left(1-\beta_{1} * \exp ^{-\beta_{2} * t_{i j}}\right)^{3}
$$

El modelo no lineal Von Bertalanffy utilizado para el análisis de las curvas de crecimiento fue adaptado a modelo mixto con la inclusión de parámetros aleatorios, así:

$$
y_{i j}=\left(\beta_{0}+b_{0 i}\right) *\left(1-\beta_{1} * \exp ^{\left(-\beta_{2}+b_{2 i}\right) * t_{i j}}\right)^{3}+\varepsilon_{i j}
$$

Dónde:

$\mathrm{y}_{\mathrm{ij}}=$ peso corporal $(\mathrm{g})$ de la i-ésima ave en el j-ésimo tiempo;

$\mathrm{t}=$ tiempo, edad en días $\beta_{0}=$ Componente fijo del modelo y está relacionado con el peso asintótico cuando t tiende a infinito (porcentaje de madurez con respecto al peso adulto);

$\beta_{1}=$ Componente fijo del modelo, el cual se define como el parámetro de ajuste cuando $Y \neq 0$ o $t \neq 0$; $\beta_{2}=$ Componente fijo del modelo, entendido como el índice de madurez expresado como una proporción de porcentaje del máximo crecimiento con respecto al peso adulto del ave;

$b_{\text {oi }}$ y $b_{2 i}=$ efectos aleatorios asociados a los efectos fijos $\beta_{0}$ y $\beta_{2}$, definen la varianza y la covarianza de las observaciones para cada efecto fijo en la i-ésima ave;

$\mathrm{m}=$ hace referencia a la proporción del peso asintótico en que el punto de inflexión se produce;

$\varepsilon_{\mathrm{ij}}=$ Efecto residual asociado a la i-ésima ave en el j-ésimo tiempo

El residuo así como los efectos aleatorios se asumieron como independientes, con distribución normal, media cero y varianza constante.

La precisión de los modelos fue determinada por:

Correlación por el método de Pearson entre los datos observados y los predichos.

Cuadrado medio del error (CME):

$$
\mathrm{CME}=\frac{\sum_{\mathrm{i}=1}^{n}\left|\mathrm{y}_{\mathrm{i}}-\hat{\mathrm{y}}_{\mathrm{i}}\right|^{2}}{\mathrm{n}}
$$

Porcentaje de la media absoluta del error (MAPE):

$$
\text { MAPE }=\frac{\sum_{i=1}^{n}\left|\frac{y_{i}-\hat{y}_{i}}{y_{i}}\right|}{n} \times 100, \quad\left(y_{i} \neq 0\right)
$$

Media de la desviación absoluta (MAD):

$$
\mathrm{MAD}=\frac{\sum_{\mathrm{i}=1}^{n}\left|\mathrm{y}_{\mathrm{i}}-\hat{\mathrm{y}}_{\mathrm{i}}\right|}{\mathrm{n}}
$$

Para el análisis de los datos, programación de los modelos y graficación se utilizaron los paquetes lattice, neuralnet, nlme y Ime4 del software R Project (21).

\section{RESULTADOS}

EI MNL Von Bertalanffy fue altamente significativo $(p<0.001)$ y se ajustó a los datos con los valores, así:

$\mathrm{Peso}_{\mathrm{ij}}=1861.1 *\left(1-0.8131 * \exp ^{-0.0162 * \mathrm{t}_{\mathrm{ij}}}\right)^{3}+83.20$

El MNLM Von Bertalanffy, quedó estructurado con los parámetros estimados con efecto altamente significativo $(p<0.001)$, así: 
Peso $_{\text {ii }}=\left(1804.41+\left(\sigma^{2}=9.4653\right)\right) *(1-0.8144 *$

$\exp ^{\left.\left(-0.01687+\left(\sigma^{2}=0.001608\right)\right) * t_{11}\right)^{3}}+31.99$

Los disposición final de la RNA aparece en la figura 1 , donde se aprecian 19 parámetros estimados por la red ( 12 pesos de interconexión entre neuronas y 7 valores umbral).

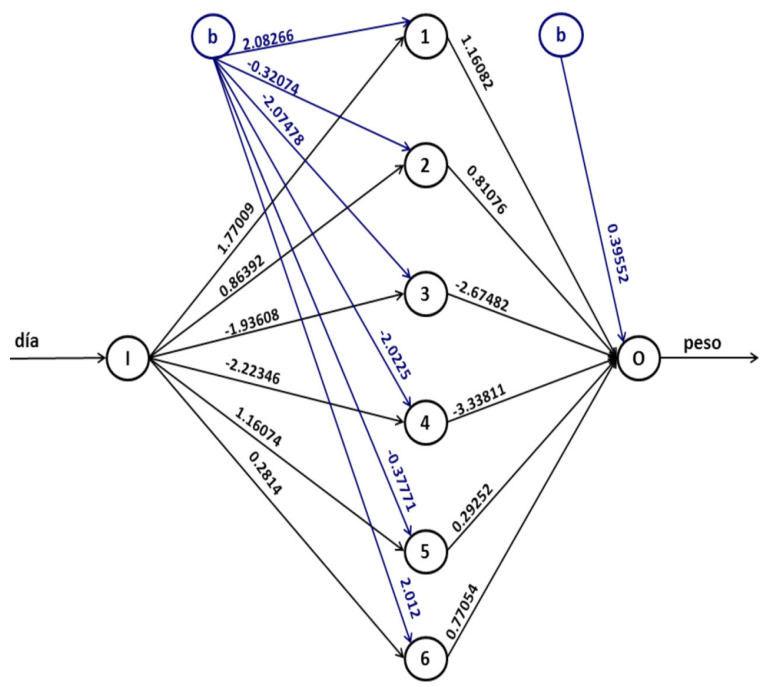

Figura 1. Estructura de la red neuronal: neurona de entrada (i) para la edad del ave en días, seis neuronas ocultas (1-6), dos neuronas de umbral (b) y una neurona de salida(o) que estima el peso del ave $(\mathrm{g})$.

En la tabla 1 se puede observar los valores de los criterios de validación y comparación de los modelos utilizados en la descripción del crecimiento de aves de la línea Lohmann LSL. El CME penaliza los modelos con mayores errores de pronóstico ya que eleva cada uno al cuadrado. El MAPE calcula los errores de pronóstico en términos de porcentaje y no de cantidades. El objetivo es obtener modelos con valores de MAPE cercanos a cero, en el caso de valores mayores a cero el modelo está subestimando los datos, mientras que valores inferiores a cero dan a entender que el modelo tiende a sobreestimar los datos. EI MAD arroja el promedio de los valores absolutos delos erros. El modelo de mejor ajuste ofrecerá menores valores de CME MAPE y MAD (22).

Tabla 1. Criterios de validación y comparación de los modelos utilizados en la descripción del crecimiento de aves de la línea Lohmann LSL.

\begin{tabular}{ccccc}
\hline & MAPE & MAD & CME & Correlación* \\
\hline MNLM & 1.964 & 11.371 & 312.61 & 0.999 \\
RNA & 7.739 & 56.284 & 4930.04 & 0.990 \\
MNL & 8.190 & 65.317 & 6921.74 & 0.986 \\
\hline
\end{tabular}

*Correlación por el método de Pearson entre el valor observado y el estimado por el modelo. Los tres modelos presentaron efecto altamente significativo $(p<0.001)$ para los valores de correlación.
EI MNLM presentó los menores valores de MAPE, MAD, CME y de correlación, seguido por los modelos RNA y MNL. Los tres modelos presentaron valores de correlación superiores al 0.98 .

Las curvas de crecimiento ajustadas por los modelos MNL, MNLM y RNA para aves de la línea Lohmann LSL aparecen en la figura 2 . Se puede ver que la línea media de predicción del MNLM y RNA tienen una forma similar, logrando ajustar la disminución en la tasa de crecimiento entre los días 110 y 140, periodo que sobreestimó el MNL.
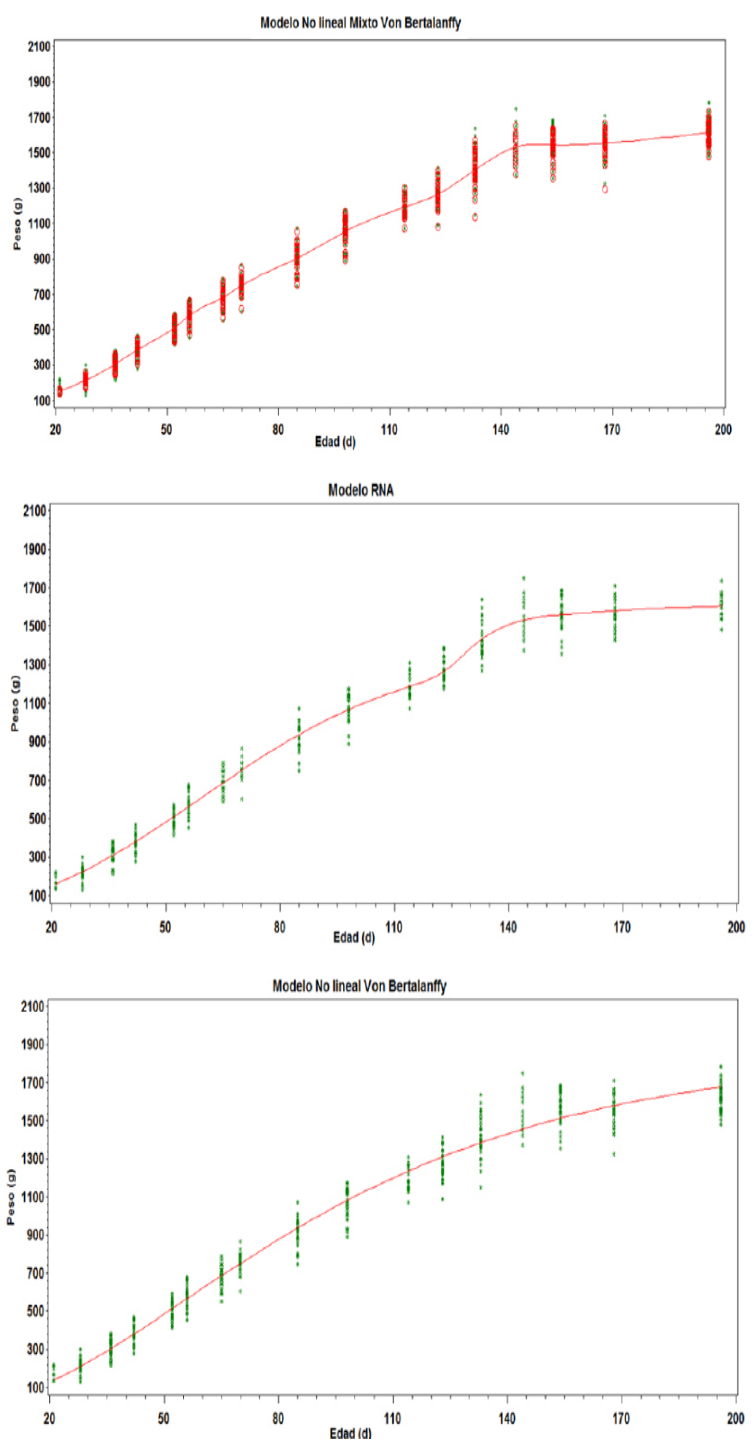

Figura 2. Curvas de crecimiento estimadas por los modelos RNA, MNLM y MNL para aves de la línea Lohmann LSL. $(0,--=$ Peso estimado por el modelo, $* * *=$ Peso real). 
Los tres modelos presentaron problemas para ajustar el inicio de la curva, donde el valor estimado fue menor al valor real, lo cual se aprecia en los gráficos de distribución de residuos (Figura 3).
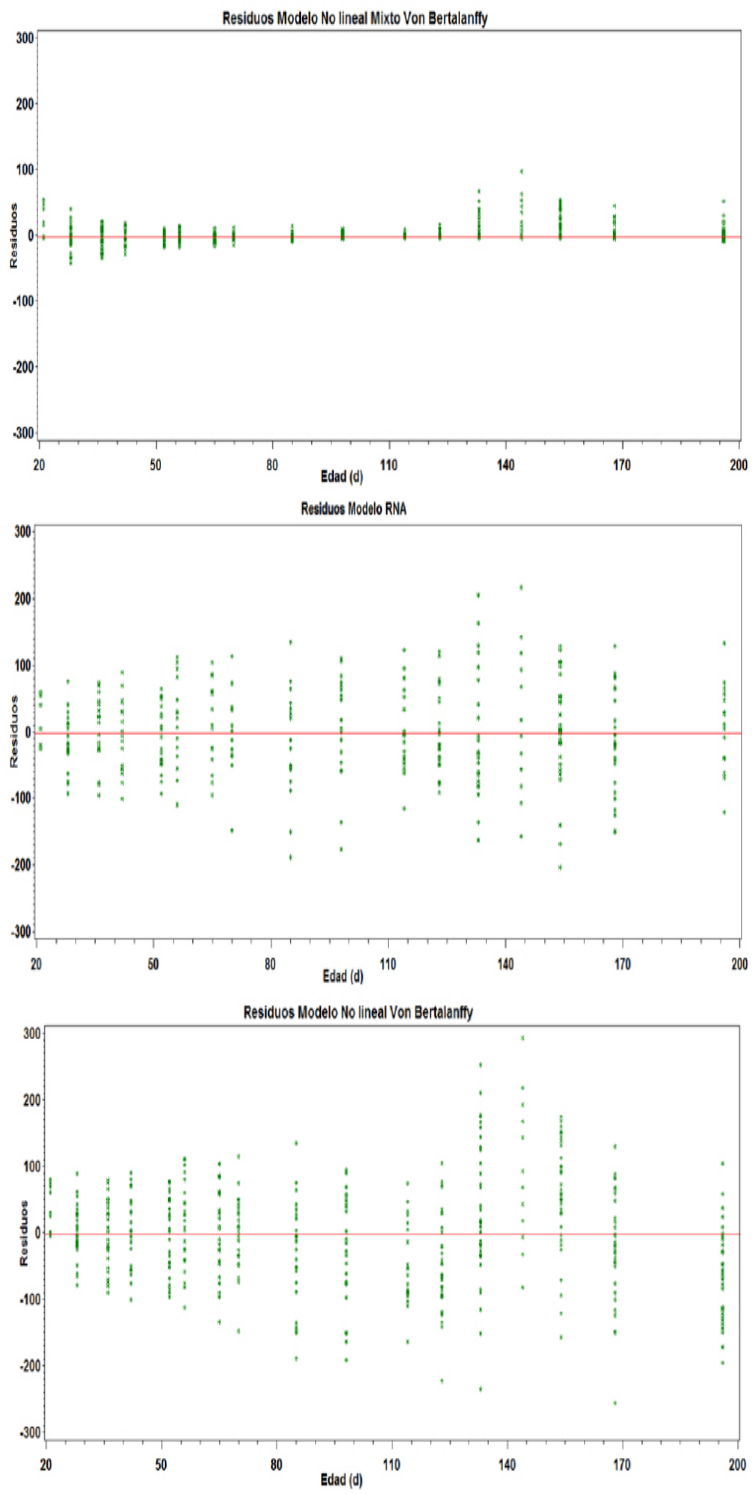

Figura 3. Distribución de residuales de los modelos utilizados en la descripción del crecimiento de aves de la línea Lohmman LSL.

El MNLM subestima los datos en 9 de las 17 mediciones, solo del día 28 al 70 realiza un ajuste óptimo. La RNA, a excepción de la medición inicial, distribuye los residuos en similar proporción por encima y debajo de cero para los puntos de medición. El MNL tiene un desempeño de los residuos similar a las RNA, pero en el periodo entre 110 y 140 días sus residuales muestran que subestima los valores ajustados.

\section{DISCUSIÓN}

Los modelos estimaron como peso asintótico $\left(\beta_{0}\right) 1861 \mathrm{~g}$. para MNL y de $1804 \mathrm{~g}$. para el MNLM. La tasa de aceleración del crecimiento $\left(\beta_{2}\right)$ para ambos modelos fue negativa. Con estos parámetros y las derivadas de los modelos podemos estimar la edad a la que el ave llegará a su máximo peso y la edad a la que alcanzará la madures sexual ( $50 \%$ de producción de huevos), siendo de importancia para la toma de decisiones en la consecución del peso objetivo de las aves en el periodo de cría y levante. En contraste, las RNA entregan un conjunto de parámetros estimados pero no tienen ninguna explicación biológica asociada al evento que se está modelando, en este caso el crecimiento de las aves.

Según los criterios de comparación MAPE, MAD, CME y Correlación el mejor modelo fue el MNLM Von Bertalanffy, el mayor ajuste a los datos reales se logra gracias a la inclusión de los componentes aleatorios ( $b_{0}$ y $b_{2}$ ), que son las varianzas aportadas por cada individuo al parámetro fijo. Así, el modelo es capaz de estimar el valor medio de la población y adicionarle la variación en el peso que cada individuo aporta en la curva (10).

En comparación con los otros modelos, la RNA presentó mayor facilidad de programación y ejecución, ya que solo es necesaria la variación heurística en el número de neuronas ocultas para lograr el ajuste, mientras que para MNL y MNLM son cuatro $\left(\beta_{0}, \beta_{1}, \beta_{2}\right.$ y $\left.\varepsilon\right)$ y $\left(\beta_{0}, \beta_{1}, \beta_{2}\right.$, b , $b_{2}$, rho y $\varepsilon$ ) siete parámetros respectivamente, con los que el investigador debe probar de forma consecutiva hasta lograr el ajuste del modelo; por lo que la velocidad y facilidad de ajuste del MNL y MNLM dependen directamente de la calidad de los parámetros iniciales, aspecto también expresado por Roush et al (14).

La disminución en la tasa de crecimiento observada entre los días 114 y 123 que el MNLM y la RNA lograron ajustar (Figura 2), está asociada con la edad de traslado a los 112 días, periodo en el cual las aves disminuyen su consumo de alimento y agua como consecuencia de la adaptación del ave al nuevo entorno social, instalaciones y equipos del galpón con las jaulas de producción. Para evitar esta disminución en el peso, se recomienda llevar las aves a la edad de traslado con un $10 \%$ más de peso al recomendado por la línea, evitando retrasos en el crecimiento del ave y un inicio de puesta tardío $(1,2)$.

En el análisis de las gráficas de residuos se puede apreciar como los modelos sub-estimaron el crecimiento del ave al día 144. Este cambio en 
la curva, que no fue ajustado por los modelos, puede estar asociado al inicio de la producción, que en promedio se dio entre los días 126 y 154 de vida.

Para el cumplimiento del objetivo de estimar de manera más precisa los cambios en el peso del ave en la fase de cría y levante, es necesario la inclusión de nuevas variables tales como iluminación (luxes), cantidad de nutrientes consumidos, volumen de agua ingerida, temperatura del agua, factores ambientales (temperatura y humedad relativa), genética y actividades de manejo, entre otras. La incorporación de todos los posibles factores que tienen efecto en el crecimiento del ave se puede hacer con las RNA, ya que de los tres es el único modelo que posibilita el desarrollo de estas modificaciones en su estructura.
En conclusión, el mejor modelo para la predicción de curvas de crecimiento fue el MNLM de Von Bertalanffy, las redes neuronales artificiales (RNA) presentaron un desempeño similar con el MNL, con la ventaja de la facilidad en su programación, rapidez en su ajuste y además, con la posibilidad de ampliar las características de la red con la inclusión de nuevas variables que influyen en el crecimiento de las ves, siendo las redes neuronales otra opción viable para la modelación en producción animal.

\section{Agradecimietnos}

Universidad de Antioquia (CODI Sostenibilidad 2013 (Código E01727) y Mediana cuantía (Código E01533) and Departamento Administrativo de Ciencia, Tecnología e Innovación (COLCIENCIAS).

\section{REFERENCIAS}

1. Reddish JM, Nestor KE, Lilburn MS. Effect of selection for growth on onset of sexual maturity in randombred and growth-selected lines of japanese quail. Poult Sci 2003; 82:187-191.

2. Amira E El-Dlebshany. The relationship between age at sexual maturity and some productive traits in local chickens strain. Egypt Poult Sci 2008; 28(4):1253-1263.

3. Dunnington EA, Siegel PB. Age and body weight at sexual maturity in female White Leghorn. Poult Sci 1984; 63:828-830.

4. Vo KV, Boone MA, Hughes BL, Knechtges JF. Effects of ambient temperature on sexual maturity. Poult Sci 1980; 59(11):2532-2537.

5. Aggrey SE. Comparison of three nonlinear and spline regression models for describing chicken growth curves. Poult Sci 2002; 81:1782-1788.

6. Aguilar C, Cortés H, Allende R. Los modelos de simulación. Una herramienta de apoyo a la gestión pecuaria. Arch Latinoam Prod Anim 2002; 10(3): 226-231.

7. Heywang BW. Effect of cooling houses for growing chickens during hot weather. Poult Sci 1947; 26(1):20-24.

8. Brody S. Bioenergetics and growth. New York: Reinhold Publishing Corporation; 1945.
9. Laird AK, Tyler SA, Barton AD. Dynamics of normal growth. Growth 1965; 29:233-248.

10. Aggrey SE. Logistic nonlinear mixed effects model for estimating growth parameters. Poult Sci 2009; 88:276-280.

11. Richards FJ. A flexible growth function for empirical use. J Exp Bot 1959; 10:290-300.

12. Von Bertalanffy L. A quantitative theory of organic growth. Hum Biol 1938; 10:181-213.

13. Roush WB, Branton SL. A Comparison of fitting growth models with a genetic algorithm and nonlinear regression. Poult Sci 2005; 84(3):494-502.

14. Roush WB, Dozier III WA, y Branton SL. Comparison of gompertz and neural network models of broiler growth. Poult Sci 2006; 85:794-797.

15. Wang Z, Zuidhof MJ. Estimation of growth parameters using a nonlinear mixed gompertz model. Poult Sci 2004; 83:847-852.

16. Ahmadi H, Golian A. Neural network model for egg production curve. J Anim Vet Adv 2008; 7(9):1168-1170.

17. Yee D, Prior MG, Florence LZ. Development of predictive models of laboratory animal growth using artificial neural networks. Comput Appl Biosci 1993; 9(5):517-22. 
18. Pitarque A, Roy JF, Ruiz JC. Redes neurales vs modelos estadísticos: Simulaciones sobre tareas de predicción y clasificación. Psicothema 1998; 19:387-400.

19. Savegnago RP, Nunes BN, Caetano $S L$, Ferraudo AS, Schmidt GS, Ledur MS, Munari DP. Comparison of logistic and neural network models to fit to the egg production curve of White Leghorn hens. Poult Sci 2011; 2011 90:705-711.

20. Pitarque A, Ruiz JC, Roy JF. 2000. Las redes neuronales como herramientas estadísticas no paramétricas de clasificación. Psicothema 2000; 12(Supl 2):459-463.
21. R Development Core Team. R: A language and environment for statistical computing. $\mathrm{R}$ Foundation for Statistical Computing, Vienna, Austria. 2008. ISBN 3-900051-07-0; (fecha de acceso 1 de enero de 2013). URL http:// www.R-project.org.

22. Oberstone J. Management Science: Concepts, Insights, and Applications. New York: West Publ. Co; 1990. 